\title{
Asymptotic Stabilization of the Heavy Top Using Controlled Lagrangians ${ }^{1}$
}

\author{
Dong Eui Chang Jerrold E. Marsden \\ Control and Dynamical Systems 107-81 \\ California Institute of Technology \\ Pasadena, CA 91125 \\ \{dchang@cds.caltech.edu, marsden@cds.caltech.edu \\ Proc. $C D C, \mathbf{3 9},(2000), 269-273$
}

\begin{abstract}
In this paper we extend the previous work on the asymptotic stabilization of pure Euler-Poincaré mechanical systems using controlled Lagrangians to the study of asymptotic stabilization of Euler-Poincaré mechanical systems such as the heavy top.
\end{abstract}

\section{Introduction}

This paper considers asymptotic stabilization of the Euler-Poincaré mechanical systems using controlled Lagrangians. Control of Euler-Poincaré mechanical systems involves both generic control theory and knowledge of the mechanical structure of the Euler-Poincaré equations (see Marsden and Ratiu [1999]). This knowledge helps us to construct Lyapunov functions by using the energy-Casimir method. We illustrate this in the example of control of the upright spinning top.

The guiding principle behind our method of controlled Lagrangians is to consider a class of control laws that yield closed-loop systems which remain in Lagrangian form. The method thus provides a natural class of energy-based Lyapunov functions whose shape can be changed by control gains. This type of energy shaping technique, combined with dissipation, allows us to achieve asymptotic stabilization. Initial work on the controlled Lagrangian method was done in a series of papers by Bloch, Leonard and Marsden [1997, 1998, 1999a,b]. Complete discussion of the basic theory is given in Bloch, Leonard and Marsden [2000a] and Bloch, Chang, Leonard and Marsden [2000a,b], illustrating such examples as an inverted pendulum on a cart and a spherical pendulum on a puck. The controlled Lagrangian method for pure Euler-Poincaré mechanical systems is addressed in detail in Bloch, Chang, Leonard, Marsden and Woolsey [2000] and Bloch,

\footnotetext{
${ }^{1}$ Research partially supported by AFOSR contract F49620-991-0190.
}

Leonard and Marsden [2000b], examples of which are a satellite with an internal rotor and an underwater vehicle with internal rotors and coincident centers of buoyancy and gravity. An alternative control method for the underwater vehicle on the Hamiltonian side is studied in Woolsey and Leonard [1999a,b]. Related work on controlled Lagrangians is Andreev, Auckly, Kapitanski, Kelkar and White [2000], and Hamberg [2000].

This paper is organized as follows. In $\S 2$ we discuss the Euler-Poincaré matching. In $\S 3$ we apply the theory to the asymptotic stabilization of the upright spinning of the heavy top.

\section{Euler-Poincaré Matching}

In this section we address the controlled Lagrangians method for the (general) Euler-Poincaré equations. The Euler-Poincaré matching conditions are found in Bloch, Leonard and Marsden [1998] for pure Euler-Poincaré equations. Here we apply the same conditions to the general Euler-Poincaré equations. See Holm, Marsden and Ratiu [1998] for more detail about Euler-Poincaré equations.

Assume that there is a left representation of Lie group $H$ on a vector space $V$. Let $G$ be an abelian Lie group on which $H$ acts trivially. Let $L: T H \times V^{*} \times T G \rightarrow \mathbb{R}$ be a $H$-invariant function. We consider the class of mechanical systems whose kinetic energy depends on $T H \times T G$ and potential energy on $V^{*}$. The left $H$ invariance of $L$ allows us to define the reduced Lagrangian $l: \mathfrak{h} \times V^{*} \times T G \rightarrow \mathbb{R}$ by $l\left(h^{-1} v_{h}, h^{-1} x, w\right)=$ $L\left(v_{h}, x, w\right)$ for $\left(v_{h}, x, w\right) \in T H \times V^{*} \times T G$ and $h \in H$ where $\mathfrak{h}$ is the Lie algebra of $H$. In coordinates

$$
\begin{aligned}
l\left(\eta^{\alpha}, x_{\alpha}, \dot{\theta}^{a}\right) & =\frac{1}{2} g_{\alpha \beta} \eta^{\alpha} \eta^{\beta}+g_{\alpha a} \eta^{\alpha} \dot{\theta}^{a}+\frac{1}{2} g_{a b} \dot{\theta}^{a} \dot{\theta}^{b} \\
& -U\left(x_{\alpha}\right)
\end{aligned}
$$

for $\left(\eta^{\alpha}, x_{\alpha}, \dot{\theta}^{a}\right) \in \mathfrak{h} \times V^{*} \times T G$. We assume that $g_{\alpha \beta}, g_{\alpha a}$ 
and $g_{a b}$ are constant and that the controls $u_{a}$ act in the $\theta^{a}$ directions. The controlled Euler-Poincaré equations are given by

$$
\begin{aligned}
\frac{d}{d t} \frac{\partial l}{\partial \eta} & =\operatorname{ad}_{\eta}^{*} \frac{\partial l}{\partial \eta}+\frac{\partial l}{\partial x} \diamond x \\
\frac{d}{d t} \frac{\partial l}{\partial \dot{\theta}} & =u
\end{aligned}
$$

with

$$
\dot{x}(t)=-\eta(t) x(t)
$$

where the bilinear map $\diamond: V \times V^{*} \rightarrow \mathfrak{h}$ is defined by

$$
\langle\eta x, v\rangle=-\langle v \diamond x, \eta\rangle
$$

for $v \in V, x \in V^{*}$ and $\eta \in \mathfrak{h}$. Choose the following form of the controlled Lagrangian $l_{\tau, \sigma, \rho}$ :

$$
\begin{aligned}
& l_{\tau, \sigma, \rho}\left(\eta^{\alpha}, x_{\alpha}, \dot{\theta}^{a}\right)=\frac{1}{2}\left(g_{\alpha \beta}-g_{\alpha a} g^{a b} g_{\beta b}+\sigma_{a b} \tau_{\alpha}^{a} \tau_{\beta}^{b}\right) \eta^{\alpha} \eta^{\beta} \\
& +\frac{1}{2} \rho_{a b}\left(\dot{\theta}^{a}+\left(g^{a c} g_{\alpha c}+\tau_{\alpha}^{a}\right) \eta^{\alpha}\right)\left(\dot{\theta}^{b}+\left(g^{b d} g_{\beta d}+\tau_{\beta}^{b}\right) \eta^{\beta}\right) \\
& -U\left(x_{\alpha}\right) .
\end{aligned}
$$

Define the controlled momentum $\tilde{J}_{a}$ conjugate to $\theta^{a}$ by

$$
\tilde{J}_{a}=\frac{\partial l_{\tau, \sigma, \rho}}{\partial \dot{\theta}^{a}}=\rho_{a b}\left(\dot{\theta}^{b}+\left(g^{b d} g_{\beta d}+\tau_{\beta}^{b}\right) \eta^{\beta}\right) .
$$

We wish to transform the equations in (2)-(4), by an appropriate feedback $u$, to the following controlled EulerPoincaré equations of $l_{\tau, \sigma, \rho}$ :

$$
\begin{aligned}
\frac{d}{d t} \frac{\partial l_{\tau, \sigma, \rho}}{\partial \eta} & =\operatorname{ad}_{\eta}^{*} \frac{\partial l_{\tau, \sigma, \rho}}{\partial \eta}+\frac{\partial l_{\tau, \sigma, \rho}}{\partial x} \diamond x \\
\frac{d}{d t} \frac{\partial l_{\tau, \sigma, \rho}}{\partial \dot{\theta}} & =u^{\text {diss }}
\end{aligned}
$$

with

$$
\dot{x}(t)=-\eta(t) x(t)
$$

The following is the the Euler-Poincaré matching conditions from Bloch, Leonard and Marsden [1999a]:

EP-1. $\tau_{\alpha}^{a}=-\sigma^{a b} g_{b \alpha}$,

EP-2. $\sigma^{a b}+\rho^{a b}=g^{a b}$.

We can then prove the following theorem along the same lines as the proof in Bloch, Leonard and Marsden $[2000 b]$.

Theorem 2.1 Under the assumptions EP-1 and EP2, the Euler-Poincaré equations in (7)-(9) coincide with the Euler-Poincaré equations in (2)-(4) with the following choice of the control $u$ :

$$
u_{a}=u_{a}^{\mathrm{cons}}+\left(g_{a b}-k_{a}^{\alpha} g_{\alpha b}\right) \rho^{b c} u_{c}^{\mathrm{diss}}
$$

where

$$
\begin{aligned}
u_{a}^{\mathrm{cons}} & =k_{a}^{\alpha}\left(c_{\alpha \delta}^{\psi} \eta^{\delta}\left(g_{\psi \beta} \eta^{\beta}+g_{\psi b} \dot{\theta}^{b}\right)-d_{\alpha \delta}^{\psi} \frac{\partial U}{\partial x_{\delta}} x_{\psi}\right), \\
k_{a}^{\alpha} & =D_{a b} \sigma^{b c} g_{c \beta} B^{\alpha \beta} \\
B_{\alpha \beta} & =g_{\alpha \beta}-g_{\alpha b} g^{a b} g_{a \beta} \\
D^{b a} & =g^{b a}+\sigma^{b c} g_{c \beta} B^{\alpha \beta} g_{\alpha e} g^{a e},
\end{aligned}
$$

where $c_{\alpha \delta}^{\psi}$ are the structure constants of the Lie algebra $\mathfrak{h}$ and $d_{\alpha \delta}^{\psi}$ are the coordinate expression of the bilinear map $\diamond: V \times V^{*} \rightarrow \mathbb{R}$.

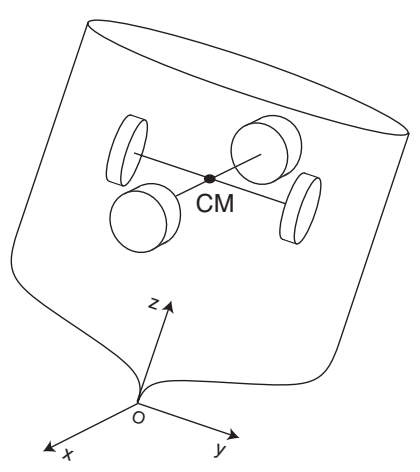

Figure 1: Heavy top with two rotors, each consisting of two rigidly coupled disks. The center of mass is at CM.

\section{Asymptotic Stabilization of the Heavy Top}

It is well-known in mechanics that the upright spinning top is unstable if the angular velocity is small. The motion of the heavy top and the stability of the Lagrange top are well studied in Marsden and Ratiu [1999] and Holm, Marsden and Ratiu [1998]. In this section we use the controlled Lagrangian method to asymptotically stabilize the upright spinning motion of the heavy top with small angular velocity, including zero velocity. Related work on the stabilization of the heavy top is Egorov and Posbergh [2000].

We first describe the heavy top with two rotors. We mount two rotors within the top so that each rotor's rotation axis is parallel to the first and the second principal axes of the top; see Figure 1 . Let $I_{1}, I_{2}, I_{3}$ be the moments of inertia of the top in the body fixed frame. Let $J_{1}, J_{2}$ be the moments of inertia of the rotors around their rotation axes. Let $J_{i 1}, J_{i 2}, J_{i 3}$ be the moments of inertia of the $i$ th rotor with $i=1,2$ around the first, the second and the third principal axes, respectively. Let $\bar{I}_{1}=I_{1}+J_{11}+J_{21}, \bar{I}_{2}=I_{2}+J_{12}+J_{22}$, and $\bar{I}_{3}=I_{3}+J_{13}+J_{23}$. Let $\lambda_{1}=\bar{I}_{1}+J_{1}$ and $\lambda_{2}=\bar{I}_{2}+J_{2}$. Let $M$ be the total mass of the system, $g$ the magnitude of the gravitational acceleration, and $h$ the dis- 
tance from the origin $O$ to the center of mass of the system.

In this example we have $H=S O(3), V^{*}=\mathbb{R}^{3}$ and $G=$ $T^{2}=S^{1} \times S^{1}$. Let $\Omega=\left(\Omega_{1}, \Omega_{2}, \Omega_{3}\right) \in \mathfrak{s o}(3) \cong \mathbb{R}^{3}$ be the angular velocity of the top in the body fixed frame. The vector $\Gamma=\left(\Gamma_{1}, \Gamma_{2}, \Gamma_{3}\right)$ represents the motion of the unit vector with the opposite direction of the gravity as seen from the body. $\theta=\left(\theta_{1}, \theta_{2}\right)$ is the rotation angle $\mathrm{s}$ of rotors around their axes. Then the reduced Lagrangian $l: \mathfrak{s o}(3) \times \mathbb{R}^{3} \times T T^{2} \rightarrow \mathbb{R}$ is given by

$$
\begin{aligned}
l(\Omega, \Gamma, \dot{\theta}) & =\frac{1}{2}\left[\begin{array}{c}
\Omega_{1} \\
\Omega_{2} \\
\Omega_{3} \\
\dot{\theta}_{1} \\
\theta_{2}
\end{array}\right]^{T}\left[\begin{array}{ccccc}
\lambda_{1} & 0 & 0 & J_{1} & 0 \\
0 & \lambda_{2} & 0 & 0 & J_{2} \\
0 & 0 & \bar{I}_{3} & 0 & 0 \\
J_{1} & 0 & 0 & J_{1} & 0 \\
0 & J_{2} & 0 & 0 & J_{2}
\end{array}\right]\left[\begin{array}{c}
\Omega_{1} \\
\Omega_{2} \\
\Omega_{3} \\
\dot{\theta}_{1} \\
\theta_{2}
\end{array}\right] \\
& -M g h \Gamma_{3} .
\end{aligned}
$$

The angular momentum $\Pi=\left(\Pi_{1}, \Pi_{2}, \Pi_{3}\right)$ is

$$
\Pi=\frac{\partial l}{\partial \Omega}=\left(\lambda_{1} \Omega_{1}+J_{1} \dot{\theta}_{1}, \lambda_{2} \Omega_{2}+J_{2} \dot{\theta}_{2}, \bar{I}_{3} \Omega_{3}\right) .
$$

The equations of motion are derived from (2)-(4) as follows:

$$
\begin{aligned}
\dot{\Pi} & =\Pi \times \Omega+M g h \Gamma \times \chi \\
\dot{\Gamma} & =\Gamma \times \Omega \\
\frac{d}{d t} \frac{\partial l}{\partial \dot{\theta}_{i}} & =u_{i}
\end{aligned}
$$

for $i=1,2$ where $\chi=(0,0,1)$ and $u_{i}$ 's are the control torques acting on the rotors.

Choose the following diagonal matrix form of $\sigma_{a b}$ and $\rho_{a b}$

$$
\left[\sigma_{a b}\right]=\left[\begin{array}{cc}
J_{1} \sigma_{1} & 0 \\
0 & J_{2} \sigma_{2}
\end{array}\right], \quad\left[\rho_{a b}\right]=\left[\begin{array}{cc}
J_{1} \rho_{1} & 0 \\
0 & J_{2} \rho_{2}
\end{array}\right]
$$

In this case the two matching conditions EP-1 and EP-2 become

$$
\left[\tau_{\theta}^{a}\right]=\left[\begin{array}{ccc}
-\frac{1}{\sigma_{1}} & 0 & 0 \\
0 & -\frac{1}{\sigma_{2}} & 0
\end{array}\right]
$$

and

$$
1=\frac{1}{\sigma_{i}}+\frac{1}{\rho_{i}}
$$

for $i=1,2$. The controlled Lagrangian $l_{\tau, \sigma, \rho}$ in (5) is computed as

$$
\begin{aligned}
& l_{\tau, \sigma, \rho}(\Omega, \Gamma, \dot{\theta})=\frac{1}{2}\left(\lambda_{1}-\frac{J_{1}}{\rho_{1}}\right) \Omega_{1}^{2}+\frac{1}{2}\left(\lambda_{2}-\frac{J_{2}}{\rho_{2}}\right) \Omega_{2}^{2} \\
& +\frac{1}{2} \bar{I}_{3} \Omega_{3}^{2}+\frac{1}{2} J_{1} \rho_{1}\left(\frac{\Omega_{1}}{\rho_{1}}+\dot{\theta}_{1}\right)^{2}+\frac{1}{2} J_{2} \rho_{2}\left(\frac{\Omega_{2}}{\rho_{2}}+\dot{\theta}_{2}\right)^{2} \\
& -M g h \Gamma_{3} .
\end{aligned}
$$

The controlled momentum $\tilde{J}=\left(\tilde{J}_{1}, \tilde{J}_{2}\right)$ conjugate to $\theta=\left(\theta, \theta_{2}\right)$ is

$$
\tilde{J}_{i}=\frac{\partial l_{\tau, \sigma, \rho}}{\partial \dot{\theta}_{i}}=J_{i} \Omega_{i}+J_{i} \rho_{i} \dot{\theta}_{i}
$$

with $i=1,2$. By Theorem 2.1 we have only to find an asymptotically stabilizing controller $u_{i}^{\text {diss }}$ for the following controlled Euler-Poincaré equations:

$$
\begin{aligned}
\dot{\Pi} & =\Pi \times \Omega+M g h \Gamma \times \chi \\
\dot{\Gamma} & =\Gamma \times \Omega \\
\dot{\tilde{J}}_{1} & =u_{1}^{\text {diss }} \\
\dot{\tilde{J}}_{2} & =u_{2}^{\text {diss }}
\end{aligned}
$$

where $\Pi$ is the same as that in (11) by EP-1 and EP-2. We have two constants of motion; $\Pi \cdot \Gamma$ and $\|\Gamma\|^{2}$.

Let $\Omega(0), \Gamma(0)$ and $\dot{\theta}(0)$ with $\|\Gamma(0)\|^{2}=1$ be an initial condition with

$$
\Omega_{3}^{\circ}:=\Pi(0) \cdot \Gamma(0) / \bar{I}_{3}<\sqrt{M g h / \bar{I}_{3}} .
$$

We are interested in the equilibrium $e=\left(\Omega_{e}, \Gamma_{e}, \dot{\theta}_{e}\right)$ :

$$
\Omega_{e}=\left(0,0, \Omega_{3}^{\circ}\right), \quad \Gamma_{e}=(0,0,1), \quad \dot{\theta}_{e}=(0,0)
$$

or

$$
\Omega_{e}=\left(0,0, \Omega_{3}^{\circ}\right), \quad \Gamma_{e}=(0,0,1), \quad \dot{\tilde{J}}_{e}=(0,0)
$$

which corresponds to the upright spinning top with the rotors at rest. Notice that this equilibrium lies in the same level set of $\left(\Pi \cdot \Gamma,\|\Gamma\|^{2}\right)$ as the initial condition.

We construct a Lyapunov function using the energyCasimir method (see Bloch, Chang, Leonard, Marsden, and Woolsey [2000] for more detail of this method). Set

$$
E_{\tilde{\Phi}}=K_{\tau, \sigma, \rho}+U+\Phi\left(\Pi \cdot \Gamma,\|\Gamma\|^{2}\right)+\Psi\left(\tilde{J}_{1}, \tilde{J}_{2}\right)
$$

where $U(\Gamma)=M g h \Gamma_{3}$ and $K_{\tau, \sigma, \rho}$ is given by

$$
\begin{aligned}
K_{\tau, \sigma, \rho} & =\frac{1}{2}\left(\lambda_{1}-\frac{J_{1}}{\rho_{1}}\right) \Omega_{1}^{2}+\frac{1}{2}\left(\lambda_{2}-\frac{J_{2}}{\rho_{2}}\right) \Omega_{2}^{2}+\frac{1}{2} \bar{I}_{3} \Omega_{3}^{2} \\
& +\frac{\tilde{J}_{1}^{2}}{2 J_{1} \rho_{1}}+\frac{\tilde{J}_{2}^{2}}{2 J_{2} \rho_{2}}
\end{aligned}
$$

which is the controlled kinetic energy, consisting of the quadratic terms in (15), in the new coordinates $(\Omega, \Gamma, \tilde{J})$. Choose the function $\Psi$ as follows

$$
\Psi\left(\tilde{J}_{1}, \tilde{J}_{2}\right)=\frac{\tilde{J}_{1}^{2}}{2 \epsilon_{1} J_{1}}+\frac{\tilde{J}_{2}^{2}}{2 \epsilon_{2} J_{2}}
$$

where coefficients $\epsilon_{i}$ will be determined later. Choose the function $\Phi$ of the form

$$
\begin{aligned}
\Phi(x, y) & =-\Omega_{3}^{\circ}\left(x-\bar{I}_{3} \Omega_{3}^{\circ}\right)+\frac{1}{2}\left(\bar{I}_{3}\left(\Omega_{3}^{\circ}\right)^{2}-M g h\right)(y-1) \\
& +\frac{1}{2} a_{1}\left(x-\bar{I}_{3} \Omega_{3}^{\circ}\right)^{2}+\frac{1}{2} a_{2}(y-1)^{2}
\end{aligned}
$$


where the constants $a_{1}$ and $a_{2}$ are chosen such that

$$
a_{1}<-1 / \bar{I}_{3}
$$

and

$4 a_{2}+a_{1}\left(\bar{I}_{3} \Omega_{3}^{\circ}\right)^{2}+\bar{I}_{3}\left(\Omega_{3}^{\circ}\right)^{2}-M g h<\frac{\bar{I}_{3}\left(a_{1} \bar{I}_{3} \Omega_{3}^{\circ}-\Omega_{3}^{\circ}\right)^{2}}{1+a_{1} \bar{I}_{3}}$.

One can check that the equilibrium $e$ is a critical point of $E_{\tilde{\Phi}}$. We now find conditions under which this critical point is a local maximum. First choose $\rho_{i}$ satisfying

$$
\frac{\bar{I}_{3}\left(\Omega_{3}^{\circ}\right)^{2}-M g h}{\left(\Omega_{3}^{\circ}\right)^{2}}<\lambda_{i}-\frac{J_{i}}{\rho_{i}}<0
$$

for $i=1,2$, and then we can choose $\epsilon_{1}$ and $\epsilon_{2}$ such that the second derivative of $E_{\tilde{\Phi}}$ becomes negative definite at $e$, which implies that $E_{\tilde{\Phi}}$ has a local maximum at $e$. For later use we impose an additional condition on $\rho_{i}$ and $\epsilon_{i}$ as follows:

$$
J_{i}\left(\Omega_{3}^{\circ}\right)^{2}+\left(\epsilon_{i}+\rho_{i}\right)\left(\left(\Omega_{3}^{\circ}\right)^{2}\left(\bar{I}_{3}-\lambda_{i}\right)-M g h\right) \neq 0
$$

for $i=1,2$. With (26), it is still possible to find $\rho_{i}$ and $\epsilon_{i}$ to ensure negative definiteness of the second derivative of $E_{\tilde{\Phi}}$ at $e$.

The following choice of $u^{\text {diss }}$

$$
u_{i}^{\mathrm{diss}}=c_{i}\left(\dot{\theta}_{i}+\frac{\tilde{J}_{i}}{\epsilon_{i} J_{i}}\right)
$$

with $c_{i}>0$ for $i=1,2$, implies

$$
\frac{d}{d t} E_{\tilde{\Phi}}=\sum_{i=1}^{2} c_{i}\left(\dot{\theta}_{i}+\frac{\tilde{J}_{i}}{\epsilon_{i} J_{i}}\right)^{2} \geq 0
$$

which proves the Lyapunov stability of the equilibrium $e$ in the closed-loop system. The complete control law $u$ can be obtained from Theorem 2.1.

Asymptotic stabilization will now be shown by using the LaSalle invariance principle. Since $E_{\tilde{\Phi}}$ has a local maximum at $e$, it is nondecreasing in time, and $\Pi \cdot \Gamma$ and $\|\Gamma\|^{2}$ are conserved, there is a number $c$ such that $S=$ $\left\{x \in \mathfrak{s o}(3) \times \mathbb{R}^{3} \times T T^{2} \mid E_{\tilde{\Phi}} \geq c, \Pi \cdot \Gamma=\Pi_{e} \cdot \Gamma_{e},\|\Gamma\|^{2}=\right.$ $1\}$ is compact and positively invariant. Define $\mathcal{E}=\{x \in$ $\left.S \mid \dot{E}_{\tilde{\Phi}}=0\right\}=\left\{x \in S \mid u^{\text {diss }}=0\right\}$. Let $\mathcal{M}$ be the largest invariant subset of $\mathcal{E}$. One can show $\mathcal{M}=\{e\}$ by (26) after shrinking the set $S$ if necessary. Thus, by the LaSalle invariance principle, $e$ is asymptotically stable.

Remark. The above procedure shows that the choice of control gains depends on the initial condition. This is unavoidable because we need to know the value of the constant of motion $\Pi \cdot \Gamma$ which the internal actuation cannot change; however, our suggested controller is robust to small errors in the measurement of the initial condition. Let $\tilde{e}$ be the equilibrium of the form
(22) with $\tilde{\Omega}_{3}^{\circ}$ instead of $\Omega_{3}^{\circ}$. Suppose the $\Omega_{3}^{\circ}$ used in constructing the control law is very close to the value $\tilde{\Omega}_{3}^{\circ}$. Let $\tilde{E}_{\tilde{\Phi}}$ be the function of the form (23) with $\Omega_{3}^{\circ}$ replaced by $\tilde{\Omega}_{3}^{\circ}$. Then $\tilde{e}$ is a critical point of $\tilde{E}_{\tilde{\Phi}}$. By continuity, the second derivative of $\tilde{E}_{\tilde{\Phi}}$ at $\tilde{e}$ will remain negative definite, proving Lyapunov stability of $\tilde{e}$. The condition (26) also holds by continuity, ensuring asymptotic stability of $\tilde{e}$.

Remark. The same form of controller works for the asymptotic stabilization of the upright spinning top with $\Omega_{3}^{\circ}>\sqrt{M g h / \bar{I}_{3}}$, which is the opposite of $(21)$. All that needs to be done is to choose $\rho_{i}$ and $\epsilon_{i}$ to make $E_{\tilde{\Phi}}$ have a local minimum at the equilibrium and to choose negative $c_{i}$ such that $E_{\tilde{\Phi}}$ decreases in time. The same LaSalle invariance principle argument guarantees asymptotic stability.

Acknowledgments. We thank Tony Bloch, Naomi Leonard, Tudor Ratiu, Troy Smith and Craig Woolsey for helpful comments.

\section{References}

Andreev, F., D. Auckly, , L. Kapitanski, A.G. Kelkar and W. White [2000] Matching and digital control implementation for underactuated systems. Proc, $A C C, 3934-3938$.

Bloch, A.M., D.E. Chang, N.E. Leonard and J.E. Marsden [2000a] Potential and kinetic shaping for control of underactuated mechanical systems, Proc. ACC. 3913-3917.

Bloch, A.M., D.E. Chang, N.E. Leonard and J.E. Marsden [2000b] Controlled Lagrangians and the stabilization of mechanical systems II: Potential shaping and tracking, IEEE Trans. Aut. Control, submitted.

Bloch, A.M., D.E. Chang, N.E. Leonard, J.E. Marsden and C. Woolsey [2000] Asymptotic stabilization of Euler-Poincaré mechanical systems. IFAC, Workshop, Princeton, 56-61.

Bloch, A.M., N.E. Leonard and J.E. Marsden [1997] Stabilization of mechanical systems using controlled Lagrangians, Proc. CDC 36, 2356-2361.

Bloch, A.M., N.E. Leonard and J.E. Marsden [1998] Matching and stabilization by the method of controlled Lagrangians, Proc. CDC 37, 1446-1451.

Bloch, A.M., N.E. Leonard and J.E. Marsden [1999a] Stabilization of the pendulum on a rotor arm by the method of controlled Lagrangians, Proc. of ICRA '99, IEEE, 500-505.

Bloch, A.M., N. Leonard and J.E. Marsden [1999b] Potential shaping and the method of controlled Lagrangians, Proc. $C D C$ 38, 1652-1657. 
Bloch, A.M., N.E. Leonard and J.E. Marsden [2000a] Controlled Lagrangians and the stabilization of mechanical systems I: The first matching theorem, IEEE Trans. Aut. Control, to appear.

Bloch, A.M., N. Leonard and J.E. Marsden [2000b] Controlled Lagrangians and the stabilization of Euler-Poincaré mechanical systems, Preprint.

Egorov, M. and T.A. Posbergh [2000] Asymptotic stabilization of relative equilibria with application to the heavy top, IFAC, Workshop, Princeton, 104109.

Hamberg, J. [2000] Simplified conditions for matching and for generalized matching in the theory of controlled Lagrangians, Proc. ACC, 3918-3923.

Holm, D.D., J.E. Marsden and T.S. Ratiu [1998] The Euler-Poincare equations and semidirect products with applications to continuum theories, Adv. in Math. 137, 1-81

Marsden, J.E. and T.S. Ratiu [1999] Introduction to Mechanics and Symmetry. Texts in Applied Mathematics, 17, Springer-Verlag, 1994. Second Edition, 1999.

Woolsey, C.A. and N.E. Leonard [1999a] Underwater vehicle stabilization by internal rotors, Proc. ACC, 3417-3421.

Woolsey, C.A. and N.E. Leonard [1999b] Global asymptotic stabilization of an underwater vehicle using internal rotors, Proc. CDC 38, 2527-2532. 